\title{
Person-centred care
}

\author{
Author: John Oldham ${ }^{A}$
}

At a time of unprecedented demand on health services and with providers under immense pressure to deliver highquality services 7 days a week, while at the same time finding substantial cost savings, there is a real danger of care standards and safety being compromised and patients being left feeling that the NHS has failed them. In many ways, the increased demand can be celebrated: it is the result of more people living longer with complex multiple illnesses, a deeper understanding of disease processes and extraordinary advances in medicine. Yet despite this progress, people consistently report being less involved in decisions and plans about their care than they want to be. This means there is a missed opportunity to harness the impact they can make for themselves on staying well. In this special edition of Future Hospital Journal we ask 'what is person-centred care and how can it be embedded at every level of the healthcare system?' These questions are explored at the levels of the individual consultation, service provision and system design.

It is most encouraging to see a movement of people and clinicians wanting to work together to make care more person centred. Despite NHS policy endorsing person centredness and most clinicians feeling they are or want to work in a person-centred way, the delivery of this is patchy. Patients and carers at every level, whether wanting to influence policy or just be more involved in decisions about their own care, feel frustrated by a health system in which services are fragmented, which is driven by financial performance and clinicians who are focused on 'fixing' individual health problems rather than supporting the person as a whole to achieve what is important to them. Nevertheless, it seems to me that the patient view has and is being heard more and more and is being acted upon. A lot of this is due to the improved access people have to information about disease and treatments and to a greater coverage in the media around innovation and new medical technology. Patients are no longer passive recipients of care but are becoming equal partners in decisions about their own care and how and where that care is provided.

Some 10 years ago, as part of a growing commitment to understanding the experiences and wishes of patients and carers, and reflecting on these in all aspects of its work, the Royal College of Physicians (RCP) established a Patient and Carer Network (PCN). Today the PCN has over 60 members

Author: ${ }^{A}$ member of the Patient and Carer Network, Royal College of Physicians, London, UK whose views and experiences around the delivery of care are integral to the way the RCP works.

At council, committees and project levels, patients and carers have an opportunity to help shape policy in the interests of patients, carers and the wider public. This has been a hugely positive demonstration of commitment by a prestigious organisation to ensuring a meaningful partnership between doctor and patient and a health service that delivers what is important to patients. As this sort of initiative becomes normal for more health-related organisations, it is perhaps worth examining the impact it has had on the interactions between patients and health professionals, as well as the extent to which patients have been supported to make decisions that reflect their own preferences or develop greater skills and confidence in self-managing their illnesses.

At the level of designing new models of care and new organisational frameworks, we should not be afraid to ask if patient and carer involvement is productive, valued, and makes a difference to the way physicians work and organisations operate. Is being a 'critical friend' a role clinicians and managers pay attention to and does it influence the way they work or is it just a cosy demonstration of political correctness? Such questions deserve careful consideration. As is the case with health professionals, it is not unreasonable to expect the input of patients and carers to be measured and assessed for what it adds to service provision and delivery. I hope that the contributions from PCN members to this edition of the journal will reinforce my belief that patients, carers and members of the public are indeed making a difference to the way clinicians and organisations deliver care.

Themes covered in this edition include health coaching training for clinicians, which can provide them with skills and knowledge to help patients achieve sustainable behavioural change; the legal, ethical and professional considerations arising from shared decision making; an exploration of how person-centred approaches can be applied in long-term conditions and the views of several PCN members based on their personal experiences of using the NHS and their resultant views on ways to improve services for patients. Also, in a fascinating commentary written by the leading health charity Sue Ryder, we review the impact of cultural values on the way care is provided by comparing the delivery of palliative care in Albania with the UK.

The challenges to be overcome in delivering a more personcentred healthcare system are still very considerable. These include the need to meet the expectations of patients in an over-stretched service while delivering integrated care in 
an often fragmented system, with ever increasing medical subspecialisation. As NHS leaders, physicians can help to create the conditions in which person-centred healthcare can be delivered. This means working in partnership with patients and carers to really understand what matters most to them both in terms of their own personal health goals and the way healthcare services are designed and delivered. I hope that physicians will find the following articles helpful, interesting and stimulating and that they provide a good indication of the way patient involvement in the work of the RCP is making a significant contribution towards making person-centred care a reality for everyone.

Address for correspondence: Mr John Oldham, Patient Involvement Unit, External Affairs, Royal College of Physicians, 11 St Andrews Place, Regent's Park, London NW1 4LE, UK.

Email: castlebarn@btinternet.com

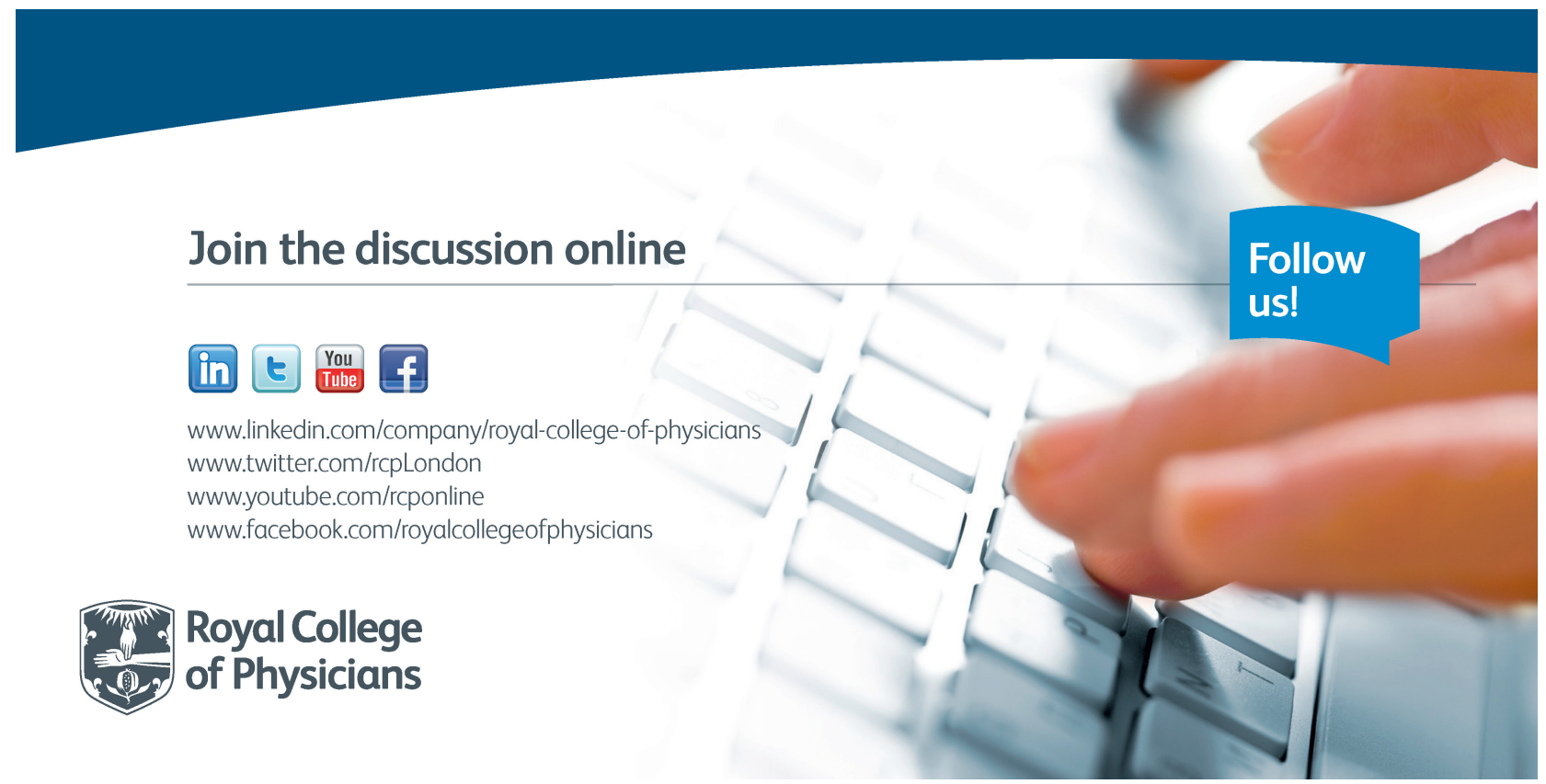

